\title{
Designing the X-ray Microcalorimeter Spectrometer for Optimal Science Return
}

\author{
Andrew Ptak ${ }^{* a}$, Simon R. Bandler ${ }^{\mathrm{a}, \mathrm{b}}$, Jay Bookbinder ${ }^{\mathrm{c}}$, Richard L. Kelley ${ }^{\mathrm{a}}$, Robert Petre ${ }^{\mathrm{a}}$, \\ Randall K. Smith ${ }^{\mathrm{c}}$, Stephen Smith ${ }^{\mathrm{a}, \mathrm{d}}$ \\ ${ }^{a}$ NASA Goddard Space Flight Center, Greenbelt, MD, USA 20771, ${ }^{\mathrm{b}}$ University of Maryland, \\ College Park, MD, USA 20742; 'Smithsonian Astrophysical Observatory, 60 Garden Street, \\ Cambridge, MA, USA 02138; ${ }^{\mathrm{d}}$ CRESST/University of Maryland, Baltimore County, Baltimore, MD \\ USA 21250, USA
}

\begin{abstract}
Recent advances in X-ray microcalorimeters enable a wide range of possible focal plane designs for the X-ray Microcalorimeter Spectrometer (XMS) instrument on the future Advanced X-ray Spectroscopic Imaging Observatory (AXSIO) or X-ray Astrophysics Probe (XAP). Small pixel designs ( $75 \mu \mathrm{m})$ oversample a 5-10" PSF by a factor of 3-6 for a $10 \mathrm{~m}$ focal length, enabling observations at both high count rates and high energy resolution. Pixel designs utilizing multiple absorbers attached to single transition-edge sensors can extend the focal plane to cover a significantly larger field of view, albeit at a cost in maximum count rate and energy resolution. Optimizing the science return for a given cost and/or complexity is therefore a non-trivial calculation that includes consideration of issues such as the mission science drivers, likely targets, mirror size, and observing efficiency. We present a range of possible designs taking these factors into account and their impacts on the science return of future large effective-area X-ray spectroscopic missions.
\end{abstract}

Keywords: X-rays, astronomy, science metrics, spectroscopy

\section{INTRODUCTION}

"New Worlds, New Horizons," (NWNH) ${ }^{1}$, the 2010 Decadal survey of Astronomy and Astrophysics report from the National Research Council, listed the highest priority science questions in the field and planned an ambitious program of space- and ground-based observatories in order to address these topics. The International X-ray observatory (IXO) ${ }^{2}$ was one of three large missions given highest priority, but the NWNH report also found that IXO's cost and the available budget known at the time did not make a mission start likely before the end of the decade. The report stated that this has the effect of

\begin{abstract}
“...allowing time for the necessary technology maturation and risk reduction. However, this situation does not diminish the committee's assessment of the importance of the discoveries that IXO would make. Because of IXO's high scientific importance, a technology development program is recommended this decade with sufficient resources - estimated to be on the order of $\$ 200$ million - to prepare IXO for favorable consideration in the next survey in 2020.” (p. 37)
\end{abstract}

In response to this report, NASA has begun active consideration of a range of less-costly X-ray missions that would maintain much of IXO's science capabilities, in addition to maintaining an active technology development program. The most mature of these designs is the Advanced X-ray Spectroscopic Imaging Observatory (AXSIO), which baselines a $10 \mathrm{~m}$ focal length mirror with $\sim 0.9 \mathrm{~m}^{2}$ of effective area at $1 \mathrm{keV}$ and a half power diameter (HPD) of 10", roughly one third of IXO's area and two times worse angular resolution (although retaining a goal of 5" HPD). The X-ray Microcalorimeter Spectrometer (XMS) detector will be the only imaging detector on AXSIO, and so it must address the science requirements on the field of view (FOV), spectral resolution, and count rate capability while also being well matched to the telescope point-spread function (PSF). In August 2013 NASA HQ issued a request for Science and Technology Definition Team members to help formulate and optimize a probe-class X-ray astrophysics mission,

*Andrew.f.Ptak@nasa.gov; phone 1301 286-1154; http://science.gsfc.nasa.gov/astrophysics/staff

UV, X-Ray, and Gamma-Ray Space Instrumentation for Astronomy XVIII, edited by

Oswald H. Siegmund, Proc. of SPIE Vol. 8859, 885903 - (c) 2013 SPIE

CCC code: $0277-786 \mathrm{X} / 13 / \$ 18 \cdot$ doi: $10.1117 / 12.2024423$

Proc. of SPIE Vol. 8859 885903-1 
hereafter the X-ray Astrophysics Probe (XAP). The starting point of the XAP study will likely be the N-CAL mission formulated in the 2011 PCOS X-ray Mission Study ${ }^{3}$. A comparison of the effective area of IXO, AXSIO and N-CAL and other missions is shown in Figure 1. The proposed calorimeter focal plane layout design parameters, identical for the current design of AXSIO and XAP, along with the IXO values for comparison, are shown in Table 1. Note that the focal length on IXO was $20 \mathrm{~m}$ and therefore the plate scale on AXSIO or XAP is a factor of two larger. This drives the overall pixel sizes to be smaller, indirectly leading to improved energy resolution. Table 1 shows the XMS parameters initially established for AXSIO in $2011^{4}$. During the PCOS X-ray study a revised array layout was proposed that would result in the same FOV but fewer TES readouts (1060 vs 1735) by once again incorporating position-sensitive 'Hydras', which enable multiple pixels to be readout with a single TES $^{5}$.

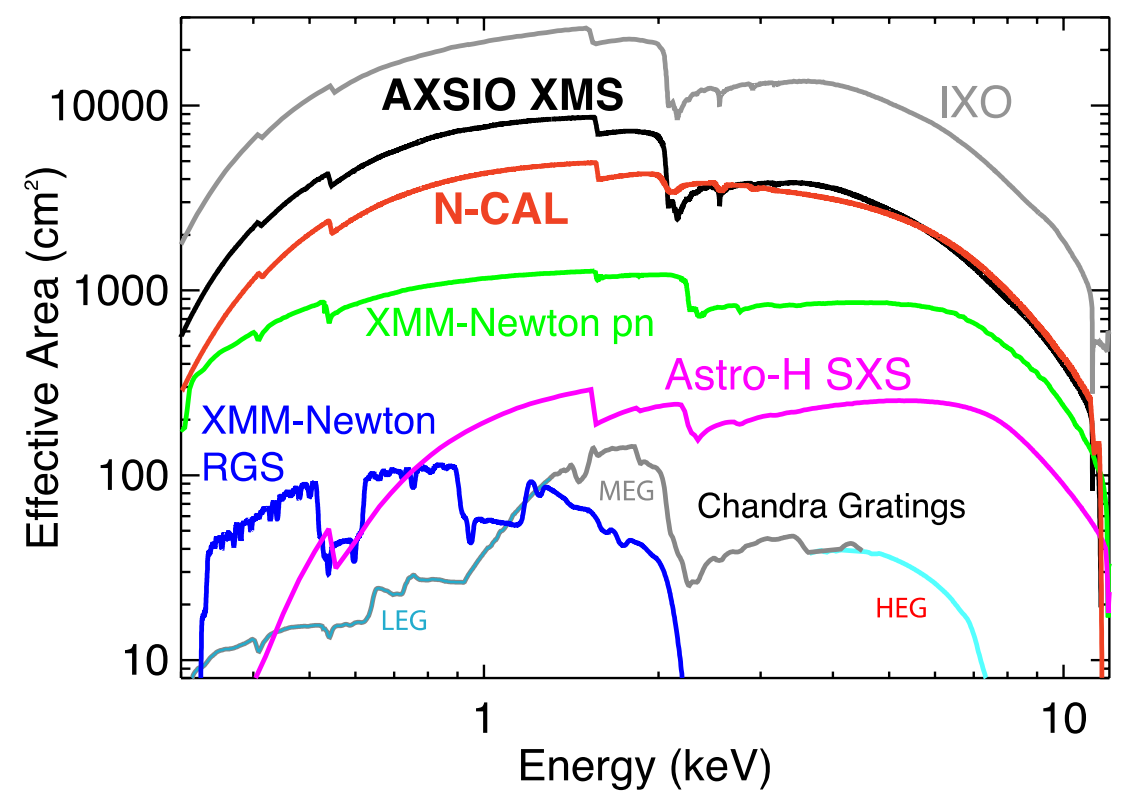

Figure 1: Comparison of the effective area of IXO, AXSIO, and N-CAL to the calorimeter on the future mission Astro-H (anticipated 2015 launch) and existing high-resolution spectrometers on Chandra and XMMNewton along with the moderate resolution XMM-Newton EPIC-pn CCD detector.

Table 1: XMS focal plane parameters for AXSIO and XAP with comparison to IXO

\begin{tabular}{|c|c|c|c|c|c|c|c|c|}
\hline \multirow{2}{*}{\multicolumn{2}{|c|}{ units }} & \multicolumn{2}{|c|}{ IXO } & \multicolumn{2}{|c|}{ AXSIO (2011) } & \multicolumn{3}{|c|}{ AXSIO/XAP } \\
\hline & & Main & Outer & $\mathrm{PSA}^{*}$ & Main & $\mathrm{PSA}^{*}$ & Main & Outer \\
\hline FOV & , & $2 \times 2$ & $5.4 \times 5.4$ & $0.3 \times 0.3$ & $4 \times 4$ & $0.4 \times 0.4$ & $1.4 \times 4.1$ & $4.1 \times 4.1$ \\
\hline Pixels & & $40 \times 40$ & $52 \times 52$ & $24 \times 24$ & $40 \times 40$ & $16 \times 16$ & $14 \times 40$ & $40 \times 40$ \\
\hline \# of TESs & & 1600 & 676 & 576 & 1564 & 256 & 544 & 260 \\
\hline Hydra factor & & $\mathrm{n} / \mathrm{a}$ & $2 \times 2$ & $\mathrm{n} / \mathrm{a}$ & $\mathrm{n} / \mathrm{a}$ & $\mathrm{n} / \mathrm{a}$ & $\mathrm{n} / \mathrm{a}$ & $2 \times 2$ \\
\hline \multirow{2}{*}{ Pixel Size } & $\mu \mathrm{m}$ & 300 & 600 & 75 & 300 & 75 & 300 & 300 \\
\hline & $\operatorname{arcsec}$ & 3 & 6 & 1.6 & 6 & 1.6 & 6 & 6 \\
\hline Resolution & $\mathrm{eV}$ & 2.5 & 10 & 2 & 3 & 2 & 3 & 6 \\
\hline Max Rate** & $\mathrm{cts} / \mathrm{s}$ & 50 & 10 & 300 & 50 & 300 & 50 & 10 \\
\hline
\end{tabular}

* Point Source Array; ** Maximum count rate at $80 \%$ throughput

\subsection{Calorimeter Design Considerations}

The AXSIO/XAP XMS design maintains the inner/outer arrangement used in IXO, but for a different reason. In IXO, the goal was to maintain at least a $5 \times 5 \operatorname{arcmin}^{2} \mathrm{FOV}$ while also having a smaller array with higher count rate and spectral 
resolution capabilities. The scientific rationale for these choices was based on neutron star equation of state (EoS) and $\mathrm{Fe} \mathrm{K}$ emission line studies in the former case, and galaxy cluster/supernova remnants in the latter. IXO required the use of a diffuser to handle high-count-rate data, since the large inner pixels could handle at most 50 counts/sec. While addressing the problem, the diffuser reduced throughput by up to $70 \%$. The current XMS design takes advantage of recent advances in high-count-rate calorimeter arrays, and defines a high-resolution Point Source Array (PSA) that covers $25 \times 25 \operatorname{arcsec}^{2}$ with a high count rate capability. With recent advances the spectral resolution is improved as well, from IXO's $2.5 \mathrm{eV}$ requirement to $2 \mathrm{eV}$ here.

The main motivation for having the outer array remains extended source science. Since the complexity and the cost scale with the number of TESs and readouts, the $2011 \mathrm{X}$-ray study team worked to reduce the number of readouts while maintaining the FOV size. This resulted in dividing the outer array into two sections as shown in Figure 2. A 1.4' x 4.1' main array uses $300 \mu \mathrm{m}$ pixels without Hydras to maintain at least $3 \mathrm{eV}$ spectral resolution. The outer array uses Hydras to maximize the FOV size but as a trade-off has a $6 \mathrm{eV}$ spectral resolution requirement.

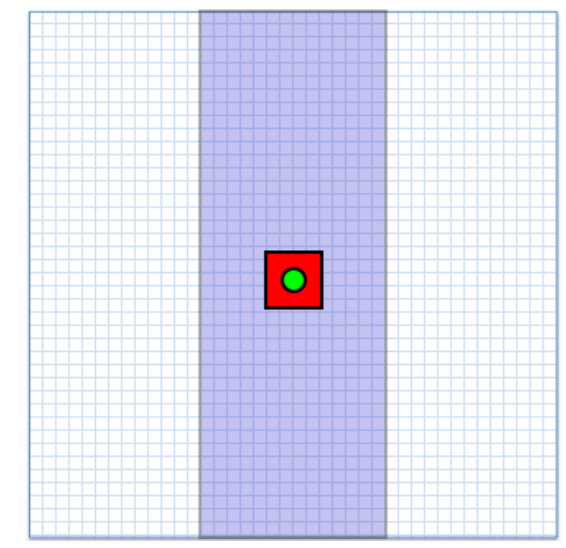

Figure 2: Nominal XMS design, with central array in red and the main array is shaded. A 10 arcsec HPD PSF shown in green.

\subsection{Mission Design Impacts of the Baseline Design}

One immediate consequence of the PSA is that AXSIO or XAP would have to improve the pointing requirement from IXO, as a bright source will need to be centered on the detector to make maximal use of the core array. It is not merely adequate to place the source on the core array, since the requirement will be to get most of the counts from the point source on the core array and (by definition) only half of the counts land within a 10 arcsec diameter circle. To ensure that at least $120 \%$ of the HPD size is on the array, the pointing requirement will have to place the detector axis to be within 6 arcsec of the desired pointing. For comparison, the IXO pointing requirement was $12 \operatorname{arcsec}$ (see RFI\#2, Table 2-6). However, in contrast to IXO, AXSIO or XAP would have likely have a fixed optical bench, which would make an improved pointing requirement very feasible.

\section{X-RAY MISSION SCIENCE OBJECTIVES}

The five key IXO science objectives that were rated highly by NWNH were:

1) What happens close to a black hole?

2) When and how did super-massive black holes (SMBH) grow?

3) How does large scale structure evolve?

4) What is the connection between SMBH formation and the evolution of large scale structure (i.e. cosmic feedback)?

5) How does matter behave at very high density? 
Since a full discussion of all possible X-ray targets would be beyond the scope of this paper, we restrict considerations to a subset of the various classes of X-ray sources, categorizing them by the expected science and the observational constraints they will impose on the mission.

At the highest level, X-ray observations can be separated into two broad classes:

Point Sources: Bright point sources are typically nearby - e.g., Galactic black holes, bursting neutron stars, nearby main sequence stars - and are also relatively rare in the X-ray sky. Although serendipitous observations are always desirable, with a FOV measured in arcminutes most point sources will not be contaminated by nearby sources. More distant point sources, such as active galactic nuclei (AGN) or ultra-luminous X-ray sources (ULX), may be embedded in diffuse emission but even so are unlikely to suffer contamination by nearby bright sources. As a result, a single PSA with size adequate to contain the full HPD will suffice to capture much of this science.

Diffuse Sources: Typical diffuse X-ray sources include galaxy clusters, hot gas in nearby galaxies, and Galactic and Local Group galaxy supernova remnants (SNRs). The primary issues are to maintain adequate energy resolution while extending the FOV to the widest possible value.

Intermediate to these two broad classes would be detecting point sources such as X-ray binaries and point-like sources such as individual supernovae HII regions in nearby galaxies. In practice, these observations would impose similar restrictions on mission design as diffuse source science.

\subsection{Spectral Resolution Science Impacts}

The use of Hydras in the outer array will reduce sensitivity to measure turbulent energy broadening and Doppler shifts. However, a $6 \mathrm{eV}$ requirement will still resolve the triplet lines from O VII and all heavier elements, and corresponds to a velocity broadening at $\mathrm{Fe} \mathrm{K}$ of $\sim 300 \mathrm{~km} / \mathrm{s}$; the detectable absolute velocity shift will be determined by the gain knowledge and not the resolution itself. This broadening value is comparable to the upper limits for cool-core clusters ${ }^{6}$ that showed that turbulent support is not significant in these clusters. These values would be considered lower limits for other clusters, since cool core clusters by definition have not had recent merger activity and are thus fairly quiescent. Although a lower value would be preferred, $6 \mathrm{eV}$ still maintains a robust science case. Reaching a $100 \mathrm{~km} / \mathrm{s}$ limit would require better than $2 \mathrm{eV}$ resolution, which would still be accomplished in the PSA and possibly also the main array.

\subsection{FOV Size Science Impacts}

All three main categories of diffuse sources that would be likely targets of a large-effective area calorimeter mission, clusters of galaxies, nearby galaxies, and SNRs, span a large range in angular extent. In the case of clusters this is shown in Figure 3, where the distribution of cluster angular sizes corresponding to a $400 \mathrm{kpc}$ radius is plotted, based on the ROSAT REFLEX cluster sample. The REFLEX sample is a flux-limited sample covering the Southern sky and therefore represents $\sim 1 / 2$ of the known clusters, and only clusters brighter than $\mathrm{F}(0.1-2.4 \mathrm{keV})=9.1 \times 10^{-12} \mathrm{ergs} \mathrm{s}^{-1} \mathrm{~cm}^{-2}$ are included in Figure 3. $400 \mathrm{kpc}$ was chosen since it is the size of the main, bright interaction region in the Bullet cluster. Figure 4 shows the XMS FOV plotted on the Bullet cluster as well as the Perseus and Coma. From these figures it is evident that with a 4' diameter FOV most clusters will require some degree of mosaicking in order to cover the entire cluster. However, as can be seen particularly in the case of the Perseus cluster, it may not be necessary to cover the entire cluster, since the most interesting regions can be placed on the PSA and the main array. 


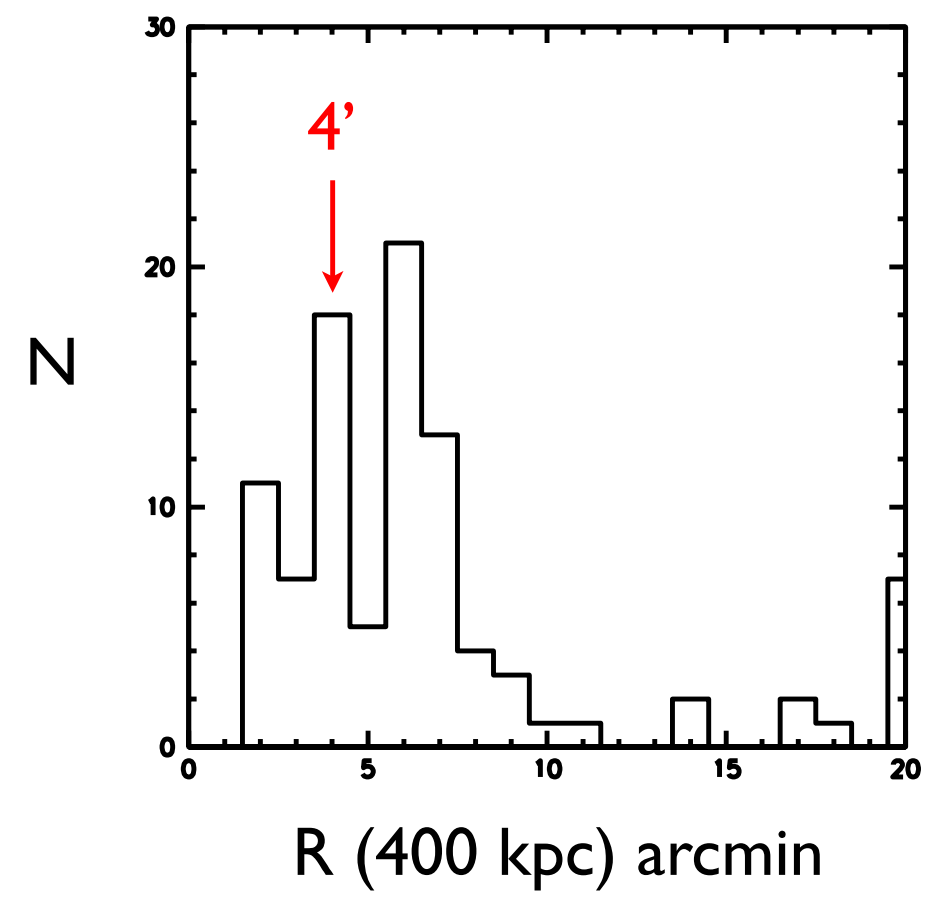

Figure 3: The distribution of cluster angular sizes corresponding to $400 \mathrm{kpc}$, based on the REFLEX catalog (M. Markevitch, p. comm). The physical size of $400 \mathrm{kpc}$ is motivated by the size of the interesting region of the Bullet cluster.

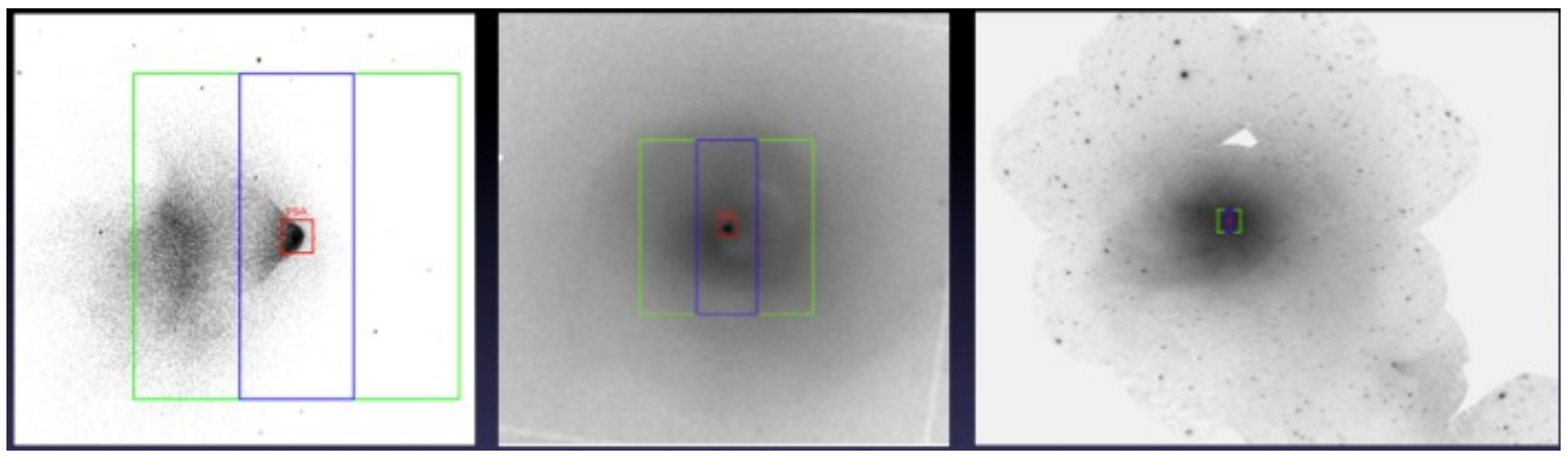

Figure 4: The XMS FOV plotted on images of the Bullet cluster (left), Perseus (middle) and the Coma cluster (right). Images courtesy of D. Wik.

In the same fashion many nearby galaxies and SNR have large angular extents, however the XMS FOV can be strategically placed to require a minimal amount of mosaicking (see Figures 5 and 6). However, this approach will obviously require roll angle constraints that in turn could limit the observing efficiency. In Figure 5 we also show the impact of blurring the Chandra image of M82 to an equivalent half-power-diameter PSF of 10". While fine details are lost in this case, as well as the ability to resolve fainter sources (with the nucleus now being clearly confused), it is also evident that the superwind outflow region is mostly free from bright "contaminating" point sources and fits fairly well within the 1.4' $\mathrm{x} 4.1^{\prime}$ ' main array where the $<3 \mathrm{eV}$ resolution calorimeter resolution would be sufficient to resolve $\sim 100$ $\mathrm{km} / \mathrm{s}$ outflow velocities (see Strickland et al. 2011 Astro2010 RFI submission, which assumed $2.5 \mathrm{eV}$ resolution). 


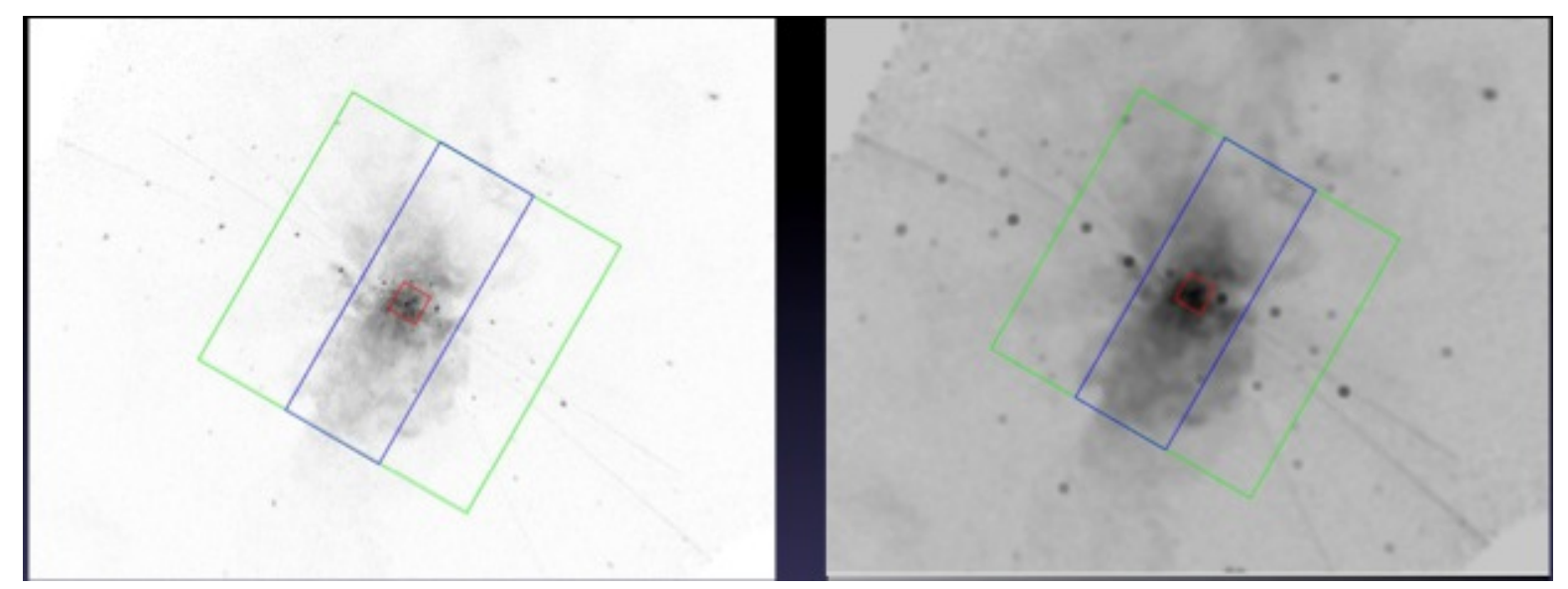

Figure 5: A Chandra image of the nearby starburst galaxy M82, with the XMS FOV plotted. The image on the right has been smoothed to a 10" HPD.
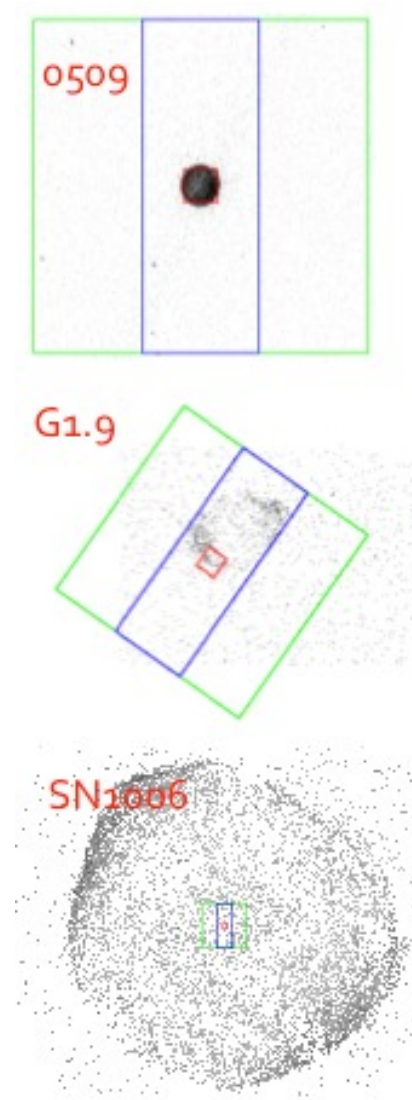
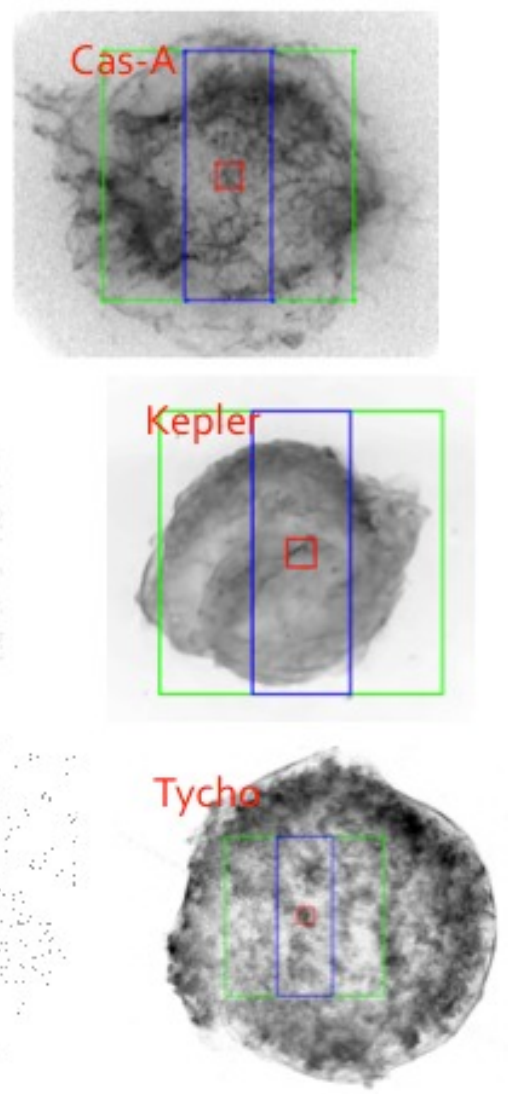
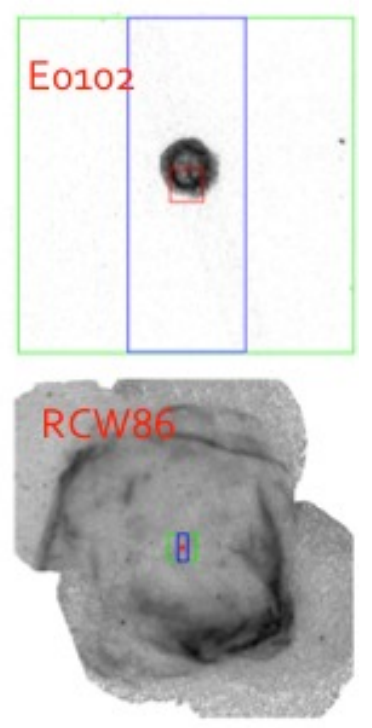

Figure 6: A collection of Chandra images of Galactic and Magellanic Cloud supernova remnants (courtesy of B. Williams), with the XMS FOV shown.

\subsection{Timing Capability}

The two main aspects of timing analysis in X-ray astronomy are the timing resolution of the detector(s) and the count rate limit of the detector(s). The relative timing resolution will depend mostly on the readout rate of the detector while the absolute timing resolution will be a function of the mission design e.g., the presence of a stable (and preferably 
atomic) clock or GPS systems to determine the orbit precisely. The current XMS timing resolution requirement of $10 \mu \mathrm{s}$ is realistic for a mission not entirely focused on timing. As stated above, a primary motivation for the PSA is to increase the bright source flux limit of the XMS without requiring a diffuser. In practice at high count rates the event quality and hence spectral resolution degrades due to multiple events occurring in a typical record length for a single triggered event, on the order of $0.5 \mathrm{~ms}$. Table 2 shows the event quality distribution from Lee et al. (2013) for a 96 counts/s source illuminating a PSA-like pixel with a $\sim 200 \mu$ s decay constant, which shows that most events were high resolution and the net spectral resolution when combining all detected photons was $2.3 \mathrm{eV}$. This strongly implies that a throughput of $80 \%$ at 300 counts/s is a realistic requirement.

Table 2: Event quality distribution for a single pixel with a $\sim 100$ count/s source from Lee et al. $(2013)^{7}$.

\begin{tabular}{|l|l|l|}
\hline Event Grade & FWHM Resolution (eV) & Throughput* \\
\hline High-res & $1.95 \pm 0.04$ & $69.0 \%$ \\
\hline Mid-res & $2.25 \pm 0.11$ & $10.1 \%$ \\
\hline Low-res & $3.78 \pm 0.08$ & $20.4 \%$ \\
\hline Combined & $2.29 \pm 0.04$ & $99.6 \%$ \\
\hline
\end{tabular}

* Fraction of photons detected at the given spectral resolution.

In practice the efficacy of a PSA for increasing the point source flux limit will depend on how well the PSF is resolved. If the spectral resolution is to be maximized at a given count rate, then the "worst-case" scenario is a point source perfectly centered on the central most pixel in the PSA, which will maximize the count rate in that pixel. Figure 7 shows a 10" and a 5" HPD PSF, the requirement and goal PSF HPD for AXSIO, on the current XMS design PSA, a 16x16 array with 1.6 " pixels. In these cases the central pixel would receive $2.0 \%$ and $7.9 \%$ of the counts. Therefore a 100 counts/s limit would translate to a 5000 or $\sim 1300$ counts/s source. For a $300 \mathrm{count} / \mathrm{s}$ limit pixel these rates would then be at least 15000 and 3900 counts/s total. For comparison, for the AXSIO effective area a 1 Crab source is expected to produce $\sim 40,000$ counts $/ \mathrm{s}$.
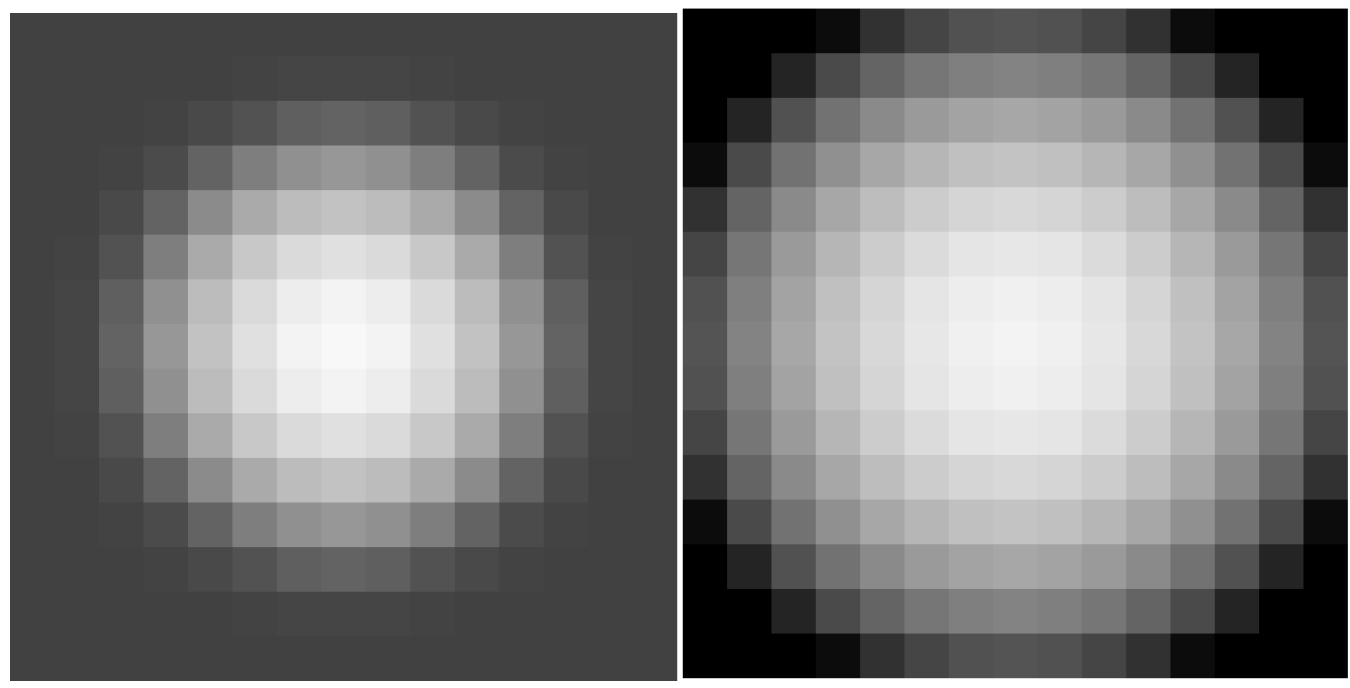

Figure 7: Model Gaussian PSFs with a 5" (left) and 10" (right) HPD, projected onto the PSA of 16x16 1.4" pixels. 


\section{MISSION DESIGN OPTIONS}

As noted above, using a PSA in the design maintains most of the IXO point source calorimeter science while potentially achieving even higher energy resolution than expected in the IXO design. Therefore, we expect that all focal plane designs will include a PSA. For the remainder of the design, the primary trades are between the number of TESs in the system, the number of pixels per TES (the 'hydra factor'), and the size of each pixel. Increasing any of these will increase the overall field of view. However, adding TESs translates directly into increased cost, as each sensor requires readout and other electronics. As discussed above, each TES has a maximum count rate that it can accept, so increasing the 'hydra factor' reduces the maximum count rate per pixel and also impacts the effective resolution of each pixel in a non-linear fashion. Finally, increasing the pixel size also negatively impacts the energy resolution. Therefore "the figure of merit" for a calorimeter focal plane would trade off spectral resolution per pixel, FOV size, and total number of TESs, and for the PSA in particular the timing / count rate limit capability.

Table 3: Detector parameters for different designs, assuming a $\sim 10 \mathrm{~m}$ focal length optic

\begin{tabular}{|c|c|c|c|c|c|c|c|c|c|c|}
\hline Design & Array & $\begin{array}{l}\text { Size X } \\
\text { (pix) }\end{array}$ & $\begin{array}{l}\text { Size Y } \\
\text { (pixels) }\end{array}$ & $\begin{array}{l}\text { Hydra } \\
\text { factor }\end{array}$ & $\begin{array}{l}\text { Resol. } \\
\text { (eV) }\end{array}$ & $\begin{array}{l}\text { Pix. Size } \\
\text { (um) }\end{array}$ & $\begin{array}{l}\text { Pix. Size } \\
\text { (") }\end{array}$ & FOV X (') & FOV Y $\left(\left(^{\prime}\right)\right.$ & $\begin{array}{l}\begin{array}{l}\text { No. of } \\
\text { TES }\end{array} \\
\end{array}$ \\
\hline \multirow[t]{3}{*}{ IXO } & 1 & 40 & 40 & 1 & 2.5 & 300 & 3.09 & 2.06 & 2.06 & 1600 \\
\hline & 2 & 52 & 52 & 2 & 10 & 600 & 6.19 & 5.36 & 5.36 & 576 \\
\hline & & & & & & & & & Total TES: & 2176 \\
\hline \multirow[t]{3}{*}{ AXSIO(2011) } & 1 & 24 & 24 & 1 & 2 & 75 & 1.55 & 0.62 & -0.62 & 576 \\
\hline & 2 & 40 & 40 & 1 & 2.5 & 300 & 6.19 & 4.13 & 4.13 & 1564 \\
\hline & & & & & & & & & Total TES: & 2140 \\
\hline \multirow[t]{2}{*}{ Athena ${ }^{8}$} & 1 & 32 & 32 & 1 & 3 & 250 & 5.16 & 2.75 & 2.75 & 1024 \\
\hline & & & & & & & & & Total TES: & 1024 \\
\hline
\end{tabular}

$\begin{array}{ccccccccccc} & 1 & 16 & 16 & 1 & 2 & 75 & 1.55 & 0.41 & 0.41 & \underline{256} \\ & 2 & 14 & 40 & 1 & 3 & 300 & 6.19 & 1.44 & 4.13 & \underline{544} \\ & 3 & 40 & 40 & 2 & 6 & 300 & 6.19 & 4.13 & 4.13 & 260 \\ & & & & & & & & & & \end{array}$

\begin{tabular}{lllllllllll}
\hline AXSIO-Wide & 1 & 16 & 16 & 1 & 2 & 75 & 1.55 & $\underline{0.41}$ & 0.41 & $\frac{256}{276}$ \\
& 2 & 14 & 80 & 2 & 4 & 300 & 6.19 & $\underline{1.44}$ & \\
& 3 & 80 & 80 & 3 & 8 & 300 & 6.19 & 8.25 & 8.25 & $\frac{587}{587}$ \\
& & & & & & & & Total TES: $\mathbf{1 1 1 9}$
\end{tabular}

There are also design considerations with the layout of the detector. Our current XMS design includes the three different pixel types discussed above: a PSA containing small pixels that oversample the PSF, a main array that uses one pixel per TES, and an outer array that uses 4 pixels per TES. From the point of view of an observer, the most natural design 
would be a symmetric detector, with a series of nested squares. Such a design would also largely eliminate the need for satellite roll angle constraints. However, the necessity of wiring of the entire focal plane might make the current asymmetric XMS design shown in Figure 2 much simpler to build and thus less costly.

Table 3 shows a list of possible designs. The number of TESs ranges from $\sim 1000$ to $\sim 2000$ while the FOV size ranges from 4' to 8'. The assessment of designs such as these will be one of the tasks for the STDT to perform, where for example the STDT may decide that sacrificing energy resolution in the outer, low-resolution array is preferred to a smaller FOV in order to reduce the amount of mosaicking for large sources.

\section{Conclusions}

Here we have laid out some of the considerations for optimizing the science return for a calorimeter-based future X-ray mission, with emphasis on an X-ray Astrophysics Probe (XAP) that could potentially start in this decade. The primary consideration is the trade between the physical size of the calorimeter array, and hence the FOV size, and the spectral resolution and timing capability of the detector. A constraint is also to minimize the number of readouts, and hence TES thermometers. An innovative feature of the most recent calorimeter focal plane designs is to incorporate not only a point-source array that oversamples the PSF to allow for bright sources, but also a main array with individual, 2-3 eV resolution pixels and an outer array with 'Hydras' that would allow multiple pixels to utilize a single TES, and a 4' diameter total FOV size. Recent calorimeter high-count rate tests imply that PSA-like calorimeter pixels will be able to meet high-count rate requirements ( 300 counts/s/pixel at $>80 \%$ throughput) that would recover a large fraction of the original IXO bright-source calorimeter science. We also showed that this design can effectively sample all of or at least the most interesting regions of nearby diffuse sources (supernova remnants, starburst galaxies and clusters of galaxies), although in all three classes there will be a significant number of targets that have larger angular extents and would require mosaicking observations. A large amount of mosaicking would limit the total number of sources that would be accessible to a mission within a given mission lifetime. Future panels such as the recently convened XAP STDT will have to consider the trades between FOV size, spectral resolution and mission lifetime in order to maximize the science return for a given total mission cost.

ACKNOWLEDGEMENTS: We acknowledge significant contributions from the NASA Goddard Integrated Design Center, Mission Design Lab and the NASA Goddard Integrated Design Center, Instrument Design Lab in evaluating the design and costing of the XMS instruments and the missions described here, and from Gerald Daelemans who directed this effort. We also gratefully acknowledge the substantial support from the NASA X-ray Study Community Science Team members: Joel Bregman (chair), Mark Bautz, David Burrows, Webster Cash, Christine Jones, Stephen Murray, Paul Plucinsky, Brian Ramsey, Ron Remillard, and Colleen Wilson-Hodge. We thank Maxim Markevich, Dan Wik, Brian Williams, and Mihoko Yukita for providing some of the data used in this paper.

\section{REFERENCES}

[1] Blandford, R. D. et al., [New Worlds, New Horizons in Astronomy and Astrophysics], National Academies Press, Washington D. C. (2010)

[2] Bookbinder, J. “The International X-ray Observatory - RFI\#1,” Astro-ph, 1001.2329 (2010)

[3] http://pcos.gsfc.nasa.gov/studies/x-ray-mission.php

[4] Bookbinder, J. "The Advanced X-ray Spectroscopic Imaging Observatory," NASA RFI Submission, 28 October 2011, <http://pcos.gsfc.nasa.gov/.../rfi/Bookbinder-Jay-RFI-NNH11ZDA018L.pdf> (21 June 2012).

[5] Smith, S.J., et al.," "Development of position-sensitive transition-edge sensor X-ray detectors", IEEE Transactions on Applied Superconductivity 19 (2009) 451-455.

[6] Sanders, J. \& Fabian, A. "Velocity width measurements of the coolest X-ray emitting material in the cores of clusters, groups and elliptical galaxies", MNRAS, 429, 2727 (2013)

[7] Lee, S.J., et al. "High Count-rate Studies of Small- pitch Transition-Edge Sensor X-ray Microcalorimeters", JLTP, submitted, (2013) 
[8] Nandra, K. “ATHENA: The Advanced Telescope for High Energy Astrophysics," Proc. Of The X-ray Universe 2011, 22 (2011)

Proc. of SPIE Vol. $8859885903-10$

Downloaded From: http://proceedings.spiedigitallibrary.org/ on 08/14/2015 Terms of Use: http://spiedigitallibrary.org/ss/TermsOfUse.aspx 